\title{
Bicêtica
}

\section{El sentido de las} enfermedades por parásitos intestinales en poblaciones americanas, identificando dilemas bioéticos
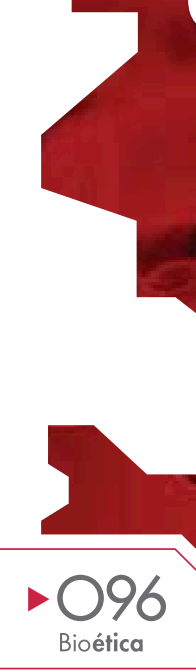

THE SENSE OF INTESTINAL PARASITIC DISEASES IN AMERICAN POPULATIONS, IDENTIFYING BIOETHICALDILEMMAS

O SETIDO DE PARASITOSES INTESTINAIS EMEPOPULAÇÕES AMERIĊANAS,

DMaría del Pilar Díaz Murillo* $\triangleright$ Nohora Aidée Ramírez Sánchez* DSamuel David Osorio García*** 


\section{RESUMEN}

Objetivo: Identificar sentidos que pueblos del continente americano le asignan a las parasitosis intestinales y plantear posibles conflictos o dilemas en las tomas de decisiones en diferentes actividades de salud producto del encuentro de valores distintos, campo de acción de la bioética. Metodología: Revisión bibliográfica de artículos sobre parasitosis intestinales que cumplieran los criterios de inclusión establecidos y suministraran aportes para la comprensión hermenéutica de las parasitosis intestinales y permitieran identificar dilemas bioéticos para actividades en salud resultantes del contacto entre las visiones émica y ética (de la biomedicina). Resultados: La construcción social de las parasitosis intestinales influye en su percepción de causalidad, nocividad y como problema de salud, lo cual afecta las acciones que la gente emprenda ante ellas. Se advierte que tales construcciones sociales pueden no coincidir con la perspectiva biomédica. Se observó que en algunas poblaciones la construcción social del cuerpo se relaciona con la percepción de efectos benéficos de los parásitos. La gente reconoce factores de riesgo pero esto no necesariamente conduce a comportamientos preventivos. Algunos estudios reconocen que la educación ha sido de tipo informativo, sin participación comunitaria, lo cual impide que la gente se identifique con ella y se sienta motivada a transformar su realidad. Se identificaron diversos dilemas bioéticos producto del contacto entre visiones de las poblaciones, y la biomedicina. Conclusiones: La aproximación émica permite entender la lógica de la población sobre las parasitosis intestinales e identificar disyuntivas bioéticas. Aunque la gente reconozca los riesgos de infección, la falta de comprensión de la perspectiva émica de los parasitismos por parte del personal de salud y las deficientes condiciones de vida permiten que no se produzcan transformaciones que incidan en la disminución de sus prevalencias. Eso mismo afectará el éxito de programas de atención, prevención, promoción y educación en la salud. Pero el análisis de la visión émica, aunque es fundamental, no lo es todo. En el problema de las parasitosis intestinales intervienen también condiciones infraestructurales, que conllevan situaciones conflictivas para los profesionales de la salud, como agentes externos a las comunidades. Se pueden hacer campañas de educación que efectivamente mejoren la prevención, pero realidades socio-económicas (por ejemplo, pobreza, participación comunitaria, empoderamiento,) y políticas (compromisos y acciones de líderes y gobiernos a diferentes niveles) pueden incidir para que lo que se aprendió no surta el efecto deseado o que se modifiquen las condiciones estructurales que generan los problemas. En este punto se generan problemas morales que deberían ser analizados por la bioética.

\section{Palabras Clave}

Antropología Médica, Parasitosis Intestinales, Promoción de la Salud, Educación en Salud, Atención Primaria de Salud.

\section{SUMMARY}

Objective: Identifying meanings that American populations assign to parasitic intestinal diseases and raising potential conflicts or dilemmas in making-decision in various health activities result of the contact between traditional and biomedical perspectives. Methodology: Bibliographic review on intestinal parasites that met the inclusion criteria and supply insights for hermeneutics understanding of intestinal parasites and allow identify bioethical dilemmas for health activities resulting from contact between the emic and ethical visions (of biomedicine). Results: The social construction of parasitic intestinal diseases influences their perception of causality, harmfulness and as a health problem. This affects actions that people undertake to these diseases. People's perception of the diseases can be different to the health professionals. Sometimes ethnoanatomy is related to beneficial effects from the parasites. People recognize risk factors but this does not necessarily lead to preventive behaviors. Some researches recognize informative education without community participation is not successful because people do not feel motivated to transform your reality. There were established various bioethical dilemmas arising from contact between emic and biomedical perspectives. Conclusions: Emic approach allows us to understand people's logic about intestinal parasites. Although people recognize infection risks from an etic perspective, health officers' lack of understanding of emic perspective of parasitic intestinal diseases, and the poor living conditions cannot produce changes that affect the reduction on prevalence rates. Those facts will affect the success of care, prevention, promotion and health education programs.

The emic perspective is not definitely. There are a lot of infrastructural conditions that developed different conflict situations for the work of the health professionals. They can provide elements for prevention and promotion strategies and programs, but depending on the socio-economical (poverty, community participation and empowerment) and political (i.e. leadership, formal commitments) conditions can be used or not. The last conclusion is that many ethical and moral problems must be analyzed by bioethics.

\section{Keywords}

Medical Anthropology, Parasitic Intestinal Diseases, Health Promotion, Health Education, Primary Health Care.

\section{RESUMO}

Objetivo: Identificar sentidos que povos das Américas atribuem às parasitoses intestinais e formular potenciais conflitos ou dilemas nas tomada de decisões em várias atividades de saúde produto do encontro de valores diferentes, o escopo da bioética. Metodologia: pesquisa de artigos sobre parasitoses intestinais que atenderam aos critérios de inclusão e forneceram contribuições para a compreensão hermenêutica das parasitoses intestinais e permitiram identificar dilemas bioéticos para atividades de saúde decorrentes do contato entre as visões émicas e éticas (da biomedicina). Resultados: A construção social das parasitoses intestinais influencia na sua percepção de causalidade, nocividade e como um problema de saúde, o que afeta as ações que as pessoas empreendem diante deles. Você é avisado de que tais construções sociais podem não corresponder à perspectiva biomédica. Observou-se que em algumas populações, a construção social do corpo está relacionada com a percepção de efeitos benéficos dos parasitas. As pessoas reconhecem fatores de risco, mas isso não conduz necessariamente a comportamentos preventivos. Alguns estudos reconhecem que a educação tem sido do tipo informativo, sem a participação da comunidade, o que impede que as pessoas se identifiquem com ela e se sinta motivada para mudar sua realidade. Identificaram-se vários dilemas bioéticos produto do contato entre as visões das populacões e da biomedicina. Conclusões: A abordagem émica permite entender a lógica das pessoas sobre os parasitas intestinais, e identificar os dilemas bioéticos. Embora as pessoas reconheçam os riscos de infecção, a falta de compreensão da perspectiva émica dos parasitismos por parte do pessoal de saúde e as condições de vida precárias permitem que não haja transformações que afetem na diminuição das suas prevalências. Isso mesmo afetará o sucesso de programas de atenção, prevenção, promoção e educação em saúde. Mas, a análise da visão émica, embora seja essencial, não é tudo. No problema das parasitoses intestinais também interferem condições de infraestrutura, que implicam situações conflitivas para os profissionais de saúde, e para agentes de fora para as comunidades. Você pode fazer campanhas de educação que efetivamente melhorem a prevenção, mas realidades socioeconômicas (por exemplo, pobreza, participação da comunidade, empoderamento) e políticas (compromissos e ações de líderes e governos em diferentes níveis) podem influenciar para que o que é se aprendeu não dei o efeito pretendido ou que se modifiquem as condições estruturais que criam os problemas. Neste momento são gerados questões morais que deveriam ser analisados pela bioética.

\section{Palavras-Chave}

Antropologia Médica, Enteropatias Parasitárias, Promoção da Saúde, Educação em Saúde, Atenção Primária à Saúde. 


\section{INTRODUCCIÓN}

Las parasitosis intestinales son un problema mundial de Salud Pública que ha causado enfermedad humana desde tiempos inmemoriales. Sus agentes causales se dividen en dos grupos: protozoarios (unicelulares) y helmintos (multicelulares) (Botero y Restrepo, 2005).

Los protozoarios intestinales patológicos que parasitan al ser humano son causa de morbilidad, malnutrición y mortalidad importantes, especialmente en niños de países en vías de desarrollo (Feng y Xiao, 2011; Stanley, 2003). La Entamoeba histolytica, agente causal de la amebiasis, causa la muerte de 40.000 - 100.000 personas cada año en todo el mundo (Lozano et al., 2012; Stanley, 2003); mientras que Giardia duodenalis es la infección intestinal por protozoos más frecuente, con un estimado de 280 millones de casos anuales a nivel mundial (Lane y Lloyd, 2002), presenta una prevalencia del 2-3\% en países industrializados y del 20-30\% en países en desarrollo (Escobedo y Cimerman, 2007).

Se estima que un billón de personas de regiones en desarrollo del África Subsahariana, América Latina y Asia, están infectadas por una o más especies de helmintos (Hotez et al., 2008; Kline, McCarthy, Pearson,

Loukas, y Hotez, 2013), de los cuales 300 millones padecen de morbilidad o deficiencia permanente grave, causando 10.000-135.000 muertes por año (Lustigman et al., 2012; OPS, 2007). Según estimaciones recientes, 1.221-1.472 millones de personas están infectadas con Ascaris lumbricoides, 795-1.050 millones con Trichuris trichiura, 7401.300 millones con Necator americanus / Ancylostoma duodenale (Utzinger y Keiser, 2004), y 30-100 millones con Strongyloides stercoralis (Olsen et al., 2009). En la mayoría de países de Latinoamérica y el Caribe hay muchas áreas con una prevalencia de helmintiasis superior a $20 \%$ (PAHO, 2009). Sin embargo el mayor impacto de las helmintiasis está en la alteración que produce en el desarrollo físico y mental de los niños, lo que a su vez afecta el desempeño escolar y la productividad económica futura (Lustigman et al., 2012; WHO, 2005).

En las Américas se estima que al menos 46 millones de niños podrían sufrir infecciones parasitarias, las cuales generan también anemia, deficiencias de vitamina A, retraso en el crecimiento, trastornos cognitivos, pérdida de memoria y disminución de la capacidad de aprender (OPS, BID, y Instituto de Vacunas Sabin, 2011).
En Latinoamérica y el Caribe el $15 \%$ de los niños en edad escolar en zonas urbanas y más de la mitad de los del área rural están en riesgo de infectarse con geohelmintos, pero sólo del 23-33\% recibe tratamiento antiparasitario (OPS et al., 2011). En Colombia, Arciniegas, Corredor y Hernández señalan que “(...) se han realizado dos encuestas nacionales de parasitismo intestinal, en 1966 y en 1980, en esta última se informan las prevalencias de A. lumbricoides 33,6 \%; T. trichiura $37,5 \%$ y Uncinaria sp. $23 \%$ en población general" (Fernández-Niño et al., 2007, p. 290). En Colombia, la última Encuesta Nacional de Morbilidad en 1980, del Instituto Nacional de Salud mostró que el $81,8 \%$ de la población tenía parásitos, porcentaje del cual $63 \%$ eran patógenos (Agudelo-Lopez et al., 2008).

El riesgo de presentar parasitosis intestinales se ve potencializado por el difícil acceso al agua potable, inadecuado saneamiento, escasos ingresos económicos de las familias y a limitaciones en el acceso a los servicios de salud (OPS et al., 2011). Por tanto son un problema relacionado con las condiciones de vida de la gente. El contexto socio-económico y cultural de estas infecciones juega un papel importante (Gamboa et al., 1998) al que no se le ha prestado suficiente atención dado el potencial impacto que tiene sobre el control de las parasitosis intestinales.

Las estadísticas sobre estas patologías, las formas de abordarlas y el tipo de educación en salud implementado corresponden comúnmente a una mirada ética (perspectiva del observador externo), la de los profesionales de la salud. Mientras la visión émica (o perspectiva de las mismas poblaciones) tiende a ser desconocida o subvalorada por los profesionales de la salud. Ello genera un vacío de conocimiento que obstaculiza la comprensión de las realidades en que están inmersas dichas patologías. En ese sentido, la visión émica faculta tanto para comprender formas de interpretar y reaccionar ante las parasitosis intestinales como para orientar las decisiones que se habrán de tomar entre el equipo de salud y las poblaciones afectadas para su control. Se debe tener en mente que la enfermedad no existe sólo como hecho biológico sino que el humano mediante su ojo cultural le atribuye sentido, la construye socialmente (Lejarraga, 2004). En consecuencia, la enfermedad existe cuando un grupo social la categoriza y explica socio-culturalmente como tal. De acuerdo a Gordon y Levin (2007) 
(...) la investigación etnográfica es (...) ideal para abrir la puerta al mundo de los significados atribuidos a los eventos sobre salud-enfermedad y las decisiones morales lque de alli se desprenden], así como para la comprensión amplia de los factores socio-económicos y políticos que moldean la forma en que las culturas y sus representantes formulan, interpretan y responden ante tales fenómenos. (p. 54. Traducción)

Las decisiones morales tienen relación directa con la Bioética, la cual se concibe

"(...) como un replanteamiento de los fundamentos que sustentan la aplicación de principios abstractos a situaciones concretas, como ha sido vista por algunos. Debe concebírsela como un replanteamiento de los fundamentos que sustentan los juicios de valor que determinan la conducta de las personas en relación con la vida, las ciencias y técnicas que la afectan, como también, en el caso de nuestro país las condiciones sociales, políticas económicas históricas educativas y culturales" (Escobar, 2009, p. 37).

El trabajo etnográfico, que da luces sobre las diferencias morales entre culturas, con una aproximación interpretativa, permite entender los razonamientos que envuelven los padecimientos. De acuerdo con Geertz (1994), tal explicación se enfoca en el significado que la gente le da, por ejemplo, a sus instituciones, costumbres y acciones. En la interpretación de las patologías hay que tener en cuenta las diferencias entre IIlness, Disease y Sickness. Para Kleinman disease (enfermedad) se refiere a una alteración o disfunción de órganos o sistemas; illness (padecimiento) se remite a la construcción cultural de la enfermedad y sickness (malestar) es el resultado de la relación entre las dos anteriores (Citado por MorenoAltamirano 2007). La última se manifiesta en el tipo de relaciones sociales a partir de valoraciones culturales. Por supuesto que el estudio del padecimiento no se reduce al sentido, pues tras cada enfermedad hay aspectos socioeconómicos y políticos (Martínez A, 2008) que facilitan ver relaciones de poder al interior de los grupos sociales.

En esa línea de pensamiento, la bioética aporta al análisis semántico social de la salud al establecer disyuntivas presentes y conflictos que adquieren nuevos significados y posiciones al darse la concurrencia de grupos sociales distintos. Como afirmara Lolas (2007), “( ...) cada vez más, los problemas del significado y las diversidades, aparentes y reales, que configuran la pluralidad de las sociedades humanas constituyen estímulos (...)" (p. 16) para buscar disciplinas que se configuren en puentes. Situación que resulta común a las ciencias humanas y sociales. La bioética es considerada una de aquellas disciplinas, la cual, de acuerdo con Potter (citado por Lolas, 2007), aporta herramientas metódicas para el análisis hermenéutico (p. 16).

Los enfoques interpretativos permiten adentrarse en las percepciones de normalidad de los padecimientos, que tienen conexión directa con la valoración socio-cultural de la enfermedad, del enfermo y de las decisiones que sobre ella y él se tomen. En este punto hay que señalar, como lo dicen Gordon y Levin (2007), que la Bioética se ubica en un punto de intersección entre el sistema moral y legal, que comprende múltiples significados, por lo cual las acciones que se tomen están cargadas simbólicamente. Por tanto, cuando saberes y morales distintas de pueblos y agentes de salud se encuentran se pueden producir "fricciones" en distintos niveles, con manifestaciones particulares, por ejemplo, desconocer al otro y realizar intervenciones verticales que no generan identificación en las comunidades.

Por otra parte, la construcción social de normalidad y de la misma enfermedad influye en su percepción y, por ende, en su expresión en la morbilidad sentida, definida por Díaz como

(...) la percepción de hechos, acciones o estados que afectan negativamente el organismo y/o las actividades habituales de los miembros de una comunidad. Cuyas manifestaciones pueden percibirse a nivel orgánico, social o psicológico, en igual o diferente proporción, dependiendo de las características del fenómeno socio-culturalmente clasificado como mórbido. (...) (Citada por Díaz Díaz, 2002, p. 39).

Si un fenómeno no merece preocupación alguna posiblemente no se actuará para controlarlo: “(...) el no buscar tratamiento no necesariamente está equivocado desde el punto de vista de los actores, especialmente si ellos consideran que los servicios de salud son irrelevantes para sus necesidades" (El Katsha y Watts, 1997).

Dado lo planteado, la antropología ayuda a evitar etnocentrismos (juicios de valor), a optimizar la forma de emplear la información, facilita la interpretación de lo que 
la gente dice y hace y permite establecer una base para la planeación y el desarrollo de estrategias y actividades de promoción de la salud, mediante claves sobre cuándo, dónde, con quién, cómo y en qué circunstancias se recomienda [o no] actuar (Krumeich, Weijts, Reddy, y Meijer-Weitz, 2001). En concordancia con lo anterior, la Bioética proporciona elementos para identificar diversos dilemas generados a partir del contacto entre visiones de las poblaciones nativas y la mirada biomédica, con el propósito de evitar imposiciones de verdades propias de los expertos a realidades de las poblaciones nativas.

En ese orden de ideas, es relevante resaltar que en 1989 el Departamento de Salud Pública de la Comunidad de Madrid (España) recomendó que el estudio integral de fenómenos de salud demanda un sistema de vigilancia

que incluya tanto la perspectiva epidemiológica como la socio-cultural (Gil Nebot, Estrada Ballesteros, Pires Alcalde, y Aguirre Martín-Gil, 2002). Entender el sentido de las patologías es importante para plantear una Educación en Salud que ayude a la concientización, la mejora en las condiciones de vida y la promoción de acciones que conduzcan a una reducción en los niveles de reinfección por parásitos intestinales.

La etnografía como método de investigación cualitativa permite entender de manera más holística los significados, creencias, y comportamientos de los grupos de poblaciones. De esta forma puede contribuir a la bioética localizando dilemas bioéticos, explicando las creencias y comportamientos de los individuos, señalando diferencias entre normas y comportamientos actuales, entre otros (Gordon y Levin, 2007). Esto se puede aplicar al contexto del estudio antropológico de las parasitosis intestinales, sacando a la luz diferentes aspectos bioéticos producto de los significados, creencias y comportamientos de las poblaciones americanas.

En este contexto, el propósito del artículo es identificar sentidos que pueblos del continente americano le asignan a las parasitosis intestinales y plantear posibles conflictos o dilemas en las tomas de decisiones en diferentes actividades de salud producto del encuentro de valores distintos, campo de acción de la bioética.

\section{METODOLOGÍA}

Se realizó una revisión bibliográfica de artículos sobre parasitosis intestinales que cumplieran todos los criterios de inclusión: publicados entre 1990 y 2011, referirse a poblaciones americanas, que contuvieran información antropológica en que se hallasen perspectivas émicas relacionadas con estas enfermedades, que estuvieran escritos en inglés, portugués o español y se hallaran aportes para la comprensión hermenéutica de las parasitosis intestinales (la hermenéutica busca identificar comportamientos generales sobre la aparición de fenómenos sociales que suceden en un contexto temporo-espacial determinado, haciendo énfasis en su interpretación (Cárcamo, 2005)). Además, debía permitir la identificación de dilemas bioéticos para actividades en salud resultantes del contacto entre las visiones émica y ética (de la biomedicina). Se excluyeron los artículos que no cumplieran alguno de los criterios de inclusión, o cumplieran uno o más criterios de exclusión: referirse a otros continentes o parasitosis, no haber sido publicado en el período 1990 a 2011, no incluir perspectivas antropológicas, o estar en idiomas diferentes a los mencionados. La exploración se adelantó en las bases de datos: J-Stor, Cochrane, Pubmed, ScienceDirect, Google Scholar, Scielo, y Lilacs, con las palabras claves: antropología médica, conocimientos, creencias, actitudes, prácticas, percepciones y representaciones sociales, parasitosis intestinales, helmintiasis e infecciones por protozoos.

Se utilizó la técnica de análisis hermenéutica para buscar entender el sentido y la explicación que tienen las expresiones lingüísticas mediante la recuperación de la visión émica de las parasitosis intestinales de las poblaciones; para esto los artículos encontrados se organizaron en 5 categorías éticas: origen percibido de los parasitismos intestinales, concepción de cuerpo y parásitos intestinales, morbilidad sentida, prevención y educación en salud. Esta división busca mostrar diferencias, reinterpretaciones y similitudes entre las perspectivas de la gente y las de la biomedicina. El análisis permitió plantear la posible presencia de dilemas bioéticos a partir del contacto de culturas diferentes, la de las poblaciones y la de los profesionales de la salud.

\section{RESULTADOS}

Se encontraron 22 publicaciones que cumplieron todos los criterios de inclusión, y ninguno de los de exclusión. Se organizaron según las siguientes 5 categorías éticas:

\section{Origen percibido de los parásitos intestinales}

Se refiere a lo que el común de las personas reconoce como la causa de las enfermedades. Es relevante 
entender cómo se construye ese origen porque además de mostrar las lógicas sobre el modo de transmisión de las parasitosis permite identificar y relacionar el tipo de conductas preventivas que se tomen desde el saber popular o tradicional. Reconocer esa percepción es importante para los médicos porque una propuesta social en salud debe surgir de establecer un diálogo participativo, que considere la relación o integración de los distintos intereses de las personas implicadas en el mismo (Pérez Serrano, 2004).

Los diferentes pueblos cuando admiten la existencia de los parasitismos les adjudican un origen particular, en el que se advierten conocimientos propios, sincretismos o reinterpretaciones, producto de diversos contactos socio-culturales. Veamos.

En un estudio sobre las geohelmintiasis en La Virgen (Cundinamarca, Colombia) se identificaron dentro de los riesgos percibidos: la ingesta de caramelos, leche (por considerarse dulce), guayaba (por los gusanos que suele tener) y frutas en descomposición; no lavarse las manos y tomar agua sin hervir (Díaz et al., 2010). Se advierte que persisten percepciones tradicionales de riesgo mezcladas con algunas provenientes de la biomedicina.

En una investigación sobre el complejo teniasiscisticercosis en Andagoya (Colombia), en un taller en el que participaron 30 adultos, el cerdo fue considerado el causante de la teniasis humana; unos cuantos la atribuyeron a pisar los excrementos de este animal. Además, relacionaron los hábitos higiénicos inadecuados con la presencia de parásitos intestinales, pero no particularmente con la teniasis-cisticercosis (AgudeloFlorez, Restrepo, y Palacio, 2009).

En algunas etnias los parásitos intestinales no son vistos de por sí como nocivos, a menos que suceda algo que permita que éstos causen enfermedad. Es el caso de los indígenas Mbya Guaraní de la provincia de Misiones (Argentina). En una revisión sobre enteroparasitosis en dos de estas comunidades Mbya se estableció que ellas consideran que los parásitos nacen con la persona y reconocen dos tipos de parásitos: "el dueño de la saliva" ubicado en la garganta y "nuestra madre parásito" localizado en la "tripa" (intestino). La segunda "produce" huevos que originan "los parásitos verdes" y "los parásitos rojos". La "cría" de estos dos tipos de parásitos se relaciona con el consumo de dulces o carne. Los "rojos" son los que en ciertas situaciones (por ejemplo, estar embarazada y comer cerdo) desencadenan la enfermedad parasitaria" (Sy, 2009). Asimismo, población
El riesgo de presentar parasitosis intestinales se ve potencializado por el difícil acceso al agua potable, inadecuado saneamiento, escasos ingresos económicos de las familias y a limitaciones en el acceso a los servicios de salud. Por tanto son un problema relacionado con las condiciones de vida de la gente. El contexto socio-económico y cultural de estas infecciones juega un papel importante al que no se le ha prestado suficiente atención dado el potencial impacto que tiene sobre el control de las parasitosis intestinales. 
chinanteca, en México, considera que la luna creciente u otros fenómenos naturales ocasionan que "los bichos" se agiten, generando desequilibrios gastrointestinales, reflejados en las diarreas (Sesia, 1999). Estos dos casos plantean disyuntivas bioéticas al momento de tomar decisiones: ¿cómo la biomedicina puede proponer estrategias preventivas para favorecer la salud de la gente, bajo la premisa del respeto al Otro, dado que hay pueblos que consideran que la gente nace con los parásitos? ¿O que la asociación percibida entre naturaleza y parasitosis intestinales muestra una visión distinta de la naturaleza a como se plantea en la historia natural de la enfermedad?

En diferentes países la gente distingue alguna relación entre higiene deficiente y parasitosis intestinales. Por ejemplo, en un estudio sobre conocimientos, actitudes y percepciones sobre las helmintiasis intestinales en Brasil la mayoría de los entrevistados reconocieron como fuentes de infección las que indica la biomedicina (tierra, alimentos sucios, suciedad, pies y boca). Un 5,5\% (48 entrevistados) afirmó que las heces eran la vía de eliminación de los gusanos, pero no supieron identificar las formas que infectan al ser humano (el huevo y/o la larva). Un 38,6\% (34 personas) no supo si existían vías de eliminación y un 75\% (66 individuos) no relacionó la eliminación de los gusanos con la contaminación ambiental, ni planteó la contaminación fecal-suelo-enfermedad. Los investigadores concluyeron que la población presenta confusiones y contradicciones respecto a los mecanismos de transmisión de las helmintiasis intestinales, sus fuentes de infección y la contaminación ambiental. Agregaron que, aunque las personas mostraron habilidades para reconocer los signos que producen los parásitos intestinales, las medidas preventivas son de difícil ejecución, pues se basan en textos foráneos que la población rechaza (Pedrazzani, Mello, Pizzigatti, y Barbosa, 1990). Algunas veces la información y respuestas ajenas provocan resistencia en los nativos, bien sea por un carácter impositivo o porque implica un cambio en sus tradiciones y creencias. Igualmente, pueden desencadenar desconfianza y dudas sobre el significado del mensaje educativo.

La infección también se la asocia con malos olores. En Popayán (Colombia) en un estudio con 24 padres de niños menores de 10 años se encontró que no identificaron formas de transmisión desde la perspectiva biomédica, pero sí un vínculo entre la infección y el hecho de vivir "...cerca del caño (...), [cuyo] olor es muy feo"
(Castro, Caldas, Flórez, Motta, y Vargas, 2008). La higiene también apareció vinculada a la giardiasis en Cuba en el que entrevistados preferían evitar compartir loza y cubiertos, entrar en contacto con la saliva de una persona infectada y malas prácticas higiénicas (como no tapar la comida) (Escobedo et al., 2011).

Se advierte que el origen de las parasitosis intestinales está socio-culturalmente construido y no existe homogeneidad en los saberes populares y tradicionales. Esto implica que los servicios de salud deben tener en cuenta la perspectiva émica en la implementación de programas de control.

\section{Concepción de cuerpo y parásitos intestinales}

La construcción sociocultural del cuerpo está relacionada con la interpretación de los efectos de los parásitos intestinales sobre las personas, lo cual permite exponer, por ejemplo, su articulación con los beneficios percibidos por algunas poblaciones americanas.

Migrantes nicaragüenses residentes en Costa Rica consideran que "los bichos" toman la comida antes que el cuerpo pueda usarla, o que éstos comen sangre y chupan nutrientes del intestino o del estómago de una persona, ocasionándole problemas de salud (Lind, 2009). Asimismo, en la etnofisiología de los indígenas Ojiteca (México), los "bichos" usualmente habitan el tracto digestivo, pero a diferencia de los anteriores piensan que los parásitos intestinales favorecen la digestión, a menos que factores de la naturaleza intervengan (Sesia, 1999).

En ese mismo sentido, en áreas rurales de Guatemala se identificó que las mujeres consideran que hay una bolsa especial donde viven las lombrices y que mientras estén allí ayudarán a la digestión. Sin embargo, si sucede algo que las perturbe (por ejemplo un empacho o ingesta de comida mal preparada) se producirá un alboroto de lombrices, lo cual hará que salgan del saco y viajen por todo el cuerpo ocasionando problemas de salud, como la diarrea (Pebley, Hurtado, y Goldman, 1996).

En zona rural de Dominica (Indias Occidentales) se determinó que para aproximarse al análisis de las parasitosis intestinales se debe entender su etnofisiología. Los individuos identifican que en su interior existe una "bolsa de gusanos": órgano específicamente destinado para los que provienen de la mugre. Se cree que al nacer los niños tienen vacía esa bolsa, la cual se va "Ilenando" cuando ingieren los huevos y al chupar algo sucio. Se considera que cuando el niño cumple un año los huevos de los gusanos 
ya se han incubado en la bolsa y que, incluso, quienes han recibido regularmente tratamiento mantienen cierta cantidad porque han ingerido mugre. Aunque hay aldeanos que afirman que los gusanos afectan su nutrición, en términos generales, la mayoría asegura que les son benéficos y que únicamente son peligrosos cuando se multiplican más allá de la capacidad que tiene la "bolsa de los gusanos" (Quinlan, Quinlan, y Nolan, 2002).

Los tres casos anteriores muestran que el interpretar como favorable "la función" de los parásitos constituye una lectura que no coincide con las explicaciones biomédicas sobre sus efectos, lo cual puede generar conflictos en los trabajos de prevención de la enfermedad y que debe ser identificada y analizada con las comunidades en las labores de educación en salud.

Por su parte, en Cuba, algunos cuidadores de niños creen que la giardia se puede esconder en un órgano del cuerpo (como la vesícula biliar o el hígado), lo cual dificultaría su tratamiento, y otros piensan que puede ocasionar úlcera duodenal. Igualmente, consideran que el coproparasitológico no es útil para determinar la presencia de la giardia y que se deberían utilizar otros métodos más apropiados (Escobedo et al., 2011).

\section{Morbilidad sentida relacionada con los parásitos intestinales}

La morbilidad sentida se refiere a la percepción de enfermedad durante un período dado. Usualmente es explicada en términos culturales específicos por las poblaciones, ya sea a través de síntomas, con categorías populares o tradicionales de enfermedad o con conceptos biomédicos, en los que se pueden identificar reinterpretaciones. Asimismo, a la percepción de enfermedad se articula la sensación de gravedad y urgencia con la que se cree debe ser tratado cada padecimiento.

En Popayán (Colombia), la población reconoce síntomas de las parasitosis intestinales, pero no les atribuye importancia, ya que asume la situación como un problema común y pasajero (Castro et al., 2008). Este caso evidencia el hecho que hay situaciones que culturalmente la gente no valora como relevantes, aunque la biomedicina sí lo haga, lo cual podrá generar dilemas sobre cómo trabajar en salud con tales poblaciones.

En Tamaco (cerca al río Napo, Amazonas) 73 entrevistadas reconocieron los parásitos intestinales como un problema de salud por su relación con la diarrea (Leah, 1999). En Cuba 71 cuidadores de niños también
Se advierte que el origen de las parasitosis intestinales está socioculturalmente construido y no existe homogeneidad en los saberes populares y tradicionales. Esto implica que los servicios de salud deben tener en cuenta la perspectiva émica en la implementación de programas de control. 
los reconocieron como generadores de riesgos serios para la salud (Escobedo et al., 2011). En un trabajo sobre dos comunidades Mbya en Argentina se estableció que las parasitosis intestinales son un problema sentido, de reciente preocupación, ya que lo atribuyen al contacto con la sociedad dominante, particularmente relacionado con cambios en su dieta, que en otros tiempos se basaba en plantas y frutas (Sy, 2009).

En una investigación aplicada en Chalcatzingo (Morelos, México) sobre Teania solium, en la que se aplicó

un cuestionario antes de una campaña educativa, se identificó que el $70 \%$ de los 146 participantes habían oído sobre la Tenia en humanos y aproximadamente la mitad la pudo describir. Pero menos del $3 \%$ sabía que se relacionaba con la infección en los cerdos. Sin embargo, la cisticercosis porcina era relativamente bien conocida por la experiencia que tenían con sus cerdos o los de los vecinos (Sarti et al., 1997).

Por su parte, en el estudio mencionado de Andagoya se realizó una encuesta CAP's (conocimientos, actitudes y prácticas) a los criadores de cerdo y sus familias sobre el complejo teniasis-cisticercosis en la que se identificó

\section{Todo grupo social tie-} ne prácticas preventivas para afrontar diversos problemas de salud, que bien pueden ser fruto de su conocimiento tradicional o producto de una mezcla o reinterpretación de otros saberes como el biomédico. que están familiarizados con la Tenia, saben que es un parásito del Hombre, "... aunque incorrectamente la consideran de transmisión fecal. Existe confusión frente a las características clínicas al referir síntomas inespecíficos como propios de la teniasis." (Agudelo-Florez et al., 2009, pp. 196-197). Asimismo comentan que su tratamiento es con antiparasitarios comerciales (no saben los nombres), pero admiten que también se puede tratar con remedios caseros (Agudelo-Florez et al., 2009).

\section{Prevención contra los parásitos intestinales}

Todo grupo social tiene prácticas preventivas para afrontar diversos problemas de salud, que bien pueden ser fruto de su conocimiento tradicional o producto de una mezcla o reinterpretación de otros saberes como el biomédico.

En La Virgen (Colombia) se advirtió que tanto niños como adultos reconocen cuáles son las medidas preventivas contra las geohelmintiasis (como beber agua sin hervir y no lavar los alimentos). "La purga" con remedios caseros o medicamentos alopáticos fue catalogada por varios adultos como una medida preventiva. Mientras los niños piensan que los parásitos mueren cuando salen del cuerpo por "no estar acostumbrados al aire puro" y "porque ya no tienen alimentación". Sin embargo, pasan por alto que los huevos sobreviven en el suelo, cuya estancia aquí les permite volverse infectantes (Díaz et al., 2010). Además, dadas

"(...) las similitudes en tamaño y forma que tienen los gusanos de la guayaba y los adultos de Uncinaria y Trichuris trichiura, los niños consideran que una forma de evitar la infección es abstenerse de consumir alimentos como la guayaba y el tomate, hecho que resulta contraproducente en tanto elimina o limita una fuente de alimento producida en la región. Desafortunadamente, esta percepción es compartida por los cuidadores."(Díaz et al., 2010, pp. 19-20)

En el estudio de Andagoya, sobre el complejo teniasis-cisticercosis, ninguno de los 30 participantes en un taller mencionó la mejora del saneamiento y la falta de confinamiento de los cerdos como elementos para el control de esa parasitosis. Se estableció sí que, aunque conocen los hábitos higiénicos para prevenir parasitosis, el modo adecuado de consumo de cerdo para evitar teniasis y el peligro del cerdo mal cocido, el consumo del mismo está difundido. Algunas personas 
dijeron que "(...) la carne con cisticercos es agradable y "jugosa" y que agregándole limón o caña agria (Costus spicatus) se destruyen los cisticercos" (Agudelo-Florez et al., 2009, p. 194). Ese tipo de apreciaciones sobre la carne de cerdo, que valora un supuesto mejor sabor sobre la salud, constituye un dilema en campañas de prevención y promoción, pues conocimientos distintos (biomédico - nativos) entran en conflicto.

El trabajo sobre Teania solium (en Morelos, México) mostró que aunque unos pocos participantes sabían cómo prevenirla, sólo una persona supo que los huevos de la Tenia en las heces se asociaba con la enfermedad (Sarti et al., 1997).

Por otro lado, aunque la información es importante en la prevención, no es determinante. Por eso se encuentran hechos contradictorios. En Popayán (Colombia) se identificó que la gente conoce formas de prevención desde la óptica biomédica, sin embargo los coproparasitológicos evidenciaron que un $62 \%$ de los niños muestreados presentaron parásitos intestinales. Por tanto, existe una brecha entre lo que la gente dice y lo que hace (Castro et al., 2008).

También, las valoraciones de los sujetos y las condiciones de vida facilitan la presencia e infección con parásitos. Por ejemplo, en Cuba se mencionó la falta de tiempo y la limitación de la distribución de combustible para no prevenir la giardiasis (Escobedo et al., 2011).

Elementos socio-económicos y culturales interactúan bien para aumentar o reducir la exposición a los riesgos de presentar enfermedades. Por ejemplo, en Branden, localidad situada al sur de Buenos Aires (Argentina), en un estudio sobre parasitosis intestinales, se realizaron talleres de intercambio de percepciones con docentes, padres y profesionales en los que se identificó carencia de conocimientos científicos y falta de conciencia sobre los factores de riesgo para la presencia y persistencia de los parásitos. Pero también se notó que la deficiencia en infraestructura y servicios básicos afecta la salud de niños y jóvenes, que constituyen la población más vulnerable a estas parasitosis (Zonta, Navone, y Oyhenart, 2007).

\section{Educación en salud respecto a los parásitos intestinales}

Estudios sugieren que la educación en salud debe renovarse, utilizar pedagogías lúdicas y fundamentarse en la participación comunitaria, acompañada con mejoras en el saneamiento ambiental y con un mayor acercamiento a la población desde una perspectiva émica.
En un trabajo en Arboleda (Nariño, Colombia) se estableció el impacto positivo para reducir la prevalencia de las parasitosis intestinales gracias a una combinación de quimioterapia, educación con un componente lúdico y el mejoramiento de condiciones sanitarias, logrado mediante solicitud voluntaria de letrinas por parte de las familias beneficiarias, quienes a cambio sembraron árboles de especies nativas con el objetivo, a futuro, de aumentar el caudal en una microcuenca del río Juanambú. La participación permitió la instalación de letrinas en todas las viviendas intervenidas y su correcta utilización (Sanzón, Vela, Valencia, y Montenegro, 1999). Dicha participación demanda compromiso y responsabilidad de la población para involucrarse en la solución de sus problemas.

En un trabajo educativo ambiental en La Virgen (Colombia) se observó que las actividades lúdicas ayudan a identificar problemas, hacer consciencia sobre ellos y a involucrar a la población en las alternativas de solución, sin imposición alguna. Dichas actividades son de gran ayuda con los niños, pues no responden al sistema escolar tradicional y les facilita aprender jugando y compartiendo con sus padres (Ramírez, Díaz, Reyes, y Cueca, 2011). Las actividades lúdicas centradas en escolares facilitan alcanzar la educación buscada y formar como actores reproductores de nuevos saberes y de estrategias preventivas contra las parasitosis (Ramírez et al., 2011) y otros problemas de las comunidades a los niños.

En una intervención educativa sobre Taenia solium, en México, se partió de identificar las concepciones sobre el ciclo de vida y de la trasmisión del parásito entre amas de casa, trabajadores agrícolas, carniceros, maestros, funcionarios locales y trabajadores de la salud (populares y modernos), para luego implementar la estrategia educativa y, posteriormente, evaluar el impacto de la misma en la prevalencia de Taenia solium. Los resultados mostraron que el desarrollo de la estrategia, con la participación de la comunidad, redujo las oportunidades de infección con T. solium. Los investigadores atribuyen el éxito de la estrategia a los datos socio-culturales que recolectaron al comienzo del trabajo (Sarti et al., 1997).

\section{DISCUSIÓN}

Las tres dimensiones de la enfermedad (disease, illness y sickness) representan aspectos distintos de la morbilidad, en cuyo análisis se debe tener en mente: 
1) ¿quién define el estado de salud de la persona? ¿Ella misma o el médico? 2) la severidad de la condición en relación con sus consecuencias para el individuo, 3) los aspectos temporales del problema de salud (agudo, crónico, recurrente...) y 4) consecuencias indirectas sobre la salud de la persona (le genera problemas socio-económicos o de otro tipo dado su papel en la sociedad) (Wikman, Marklund, y Alexanderson, 2005). La concepción de enfermedad se relaciona con la de normal, patológico

y riesgo. Lo normal remite a parámetros socio-culturales, con connotaciones valorativas, que permiten determinar lo que se considera sano, benéfico o perjudicial. Esto influye en su percepción y categorización. Por ejemplo, culturas de diferentes continentes perciben los parásitos como necesarios para la digestión (Hughes, 1975; Wenzel Geissler, 1998). Los parámetros de normalidad de la biomedicina no son universales; como lo afirmara Hughes, una teoría de la enfermedad entraña una teoría de la normalidad (Hughes, 1975) y hace un llamado urgente a la interculturalidad.

La construcción social sobre el origen de los parásitos tampoco es universal. Un bacteriólogo comentaba: "(...) es absurdo decirles [a los indígenas del Vichada] que reemplacen su sistema por el nuestro y que tienen que creer en nuestros patógenos que tampoco se ven. (...)" (Villadiego, 1995). Existen etnoanatomías diferentes a las de la biomedicina:
(...) "cada cultura construye la concepción de cuerpo sobre la base de una relación homológica en la cual, el macrocosmos, la organización social, la distribución de los espacios culturales y la actividad económica predominante, son reproducidos de una manera sintetizada e integral por el cuerpo o microcosmos" (Pinzón y Suárez, 1992, p. 46).

En ese orden de ideas como afirma Moreno-Altamirano, la conceptualización de salud y enfermedad requiere explicaciones "(...) sustentadas en modelos más complejos que incluyan un espectro más amplio que el molecular-subindividual-sistémico-ecológico en la dimensión biológica y el individual-social-cultural en la dimensión histórica" (2007, p. 69).

El adentrarse en los saberes de los pueblos (perspectiva émica) para entender los sentidos que ellos les atribuyen a las enfermedades constituye una base de la cual partir para la educación en salud. Sin embargo, esto no es una cuestión meramente instrumental, también es un compromiso moral del reconocimiento del otro, no para manipularlo sino para trabajar con él en pro de su bienestar, lo cual hace que la participación comunitaria sea un imperativo. Kloos (1995) enfatiza que la educación en salud debe empezar por preguntarse cuál es el significado que la comunidad le da a la enfermedad (en su caso sobre la esquistosomiasis, parasitismo no intestinal), a la vez que

\section{Las diferencias entre la perspectiva ética y émica manifiestan la necesidad de capacitar al personal de salud para entender y respetar otros saberes, para negociar interculturalmente con las poblaciones te- niendo en cuenta que "la salud de las colectividades está mediatizada por la interrelación de las conductas individuales, la estructura social y el ámbito cultural".}


la población debe participar en la selección de las intervenciones técnicas requeridas. Gazzinelli, Kloss, Marques, dos Reis y Gazinelli (2008), refiriéndose también a la esquistosomiasis, indican que una perspectiva culturalmente más sensible permite cerrar brechas entre el conocimiento local y el científico. La combinación de teoría y práctica en la educación, utilizando los conocimientos de la gente, su capacidad de acción y los aportes del saber científico (Pérez Serrano, 2004) se convierte en vehículo importante para la obtención de un propósito común dentro de las poblaciones, como lo es la mejora de las condiciones de vida.

Además, la educación forma valores y ayuda a construir visiones del mundo que suscitan formas de pensar y proceder. Por eso es un elemento central de la Promoción de la Salud, que fue definida en la Carta de Otawa como el "...proceso de capacitación de la comunidad para actuar en la mejoría de su calidad de vida y de salud..." (Citada por Góes, Focesi, Ribeiro, y L'Abbate, 2000, p. 10). Para León (2011) la promoción de la salud conlleva el deber ético de educar a la población y, agrega, que "desde la bioética de protección se insiste en integrar la responsabilidad moral con la eficacia pragmática, y ambas nos llevan a respetar la pluralidad de intereses y valores que presentan las colectividades (...)" (p.15).

En consecuencia, la educación en salud debe ser innovadora para articular las perspectivas émica y ética, lo cual se puede enmarcar en la lógica del respeto a la diferencia. Por ello, la educación lúdica en salud constituye una alternativa porque fomenta los valores del trabajo en equipo y el disfrute del mismo. Además, como lo dice Sanzón (1999) et al. “(...) la lúdica involucra al individuo como actor en su propio papel de vida pues asimila los conceptos de una manera simple que repercutirá en su propio beneficio". (p. 115). Enfoques centrados en la transmisión de información han sido criticados por no demostrar su efectividad en el cambio de comportamientos. Por ejemplo, Loza (citado por Kloos, 1995) refiriéndose a la esquistosomiasis, señala que ha habido estudios que muestran transformaciones en conocimientos y un creciente uso de la quimioterapia resultado de la influencia de mensajes televisivos, pero que en verdad no se han traducido en un cambio de comportamiento.

El enfoque informativo dentro de la educación en salud tiende a desconocer lo que la gente sabe y sus formas tradicionales de aprendizaje, pues está centrado en dar y no en comprender. Por eso Nikku (citado por Arratia, 1999) afirmó:
El paternalismo informativo, se presenta como una forma a través de [la] cual, los profesionales manipulan la información con la intención de influenciar al usuario de una forma benéfica, lo que lleva a crear condiciones para actuar, de acuerdo con aquello que ellos deciden como más conveniente. A través de esta acción, las autoridades y profesionales de la salud, crean expectativas e influyen en los estilos de vida, decisiones y conductas de los usuarios minimizando su conocimiento y limitando su participación ( $p$. 518, resaltado de los autores).

Las diferencias entre la perspectiva ética y émica manifiestan la necesidad de capacitar al personal de salud para entender y respetar otros saberes, para negociar interculturalmente con las poblaciones teniendo en cuenta que "la salud de las colectividades está mediatizada por la interrelación de las conductas individuales, la estructura social y el ámbito cultural" (Gil Nebot et al., 2002, p. 453). Reflexionar desde lo cultural y lo bioético sobre tales diferencias nos descubre no sólo como seres biológicos y espirituales sino también como seres sociales "necesitados continuamente de ser cuidados y respetados" (Asociación de Bioética de la Comunidad de Madrid, 2012). Dicha reflexión forma, educa. En tal sentido, la educación, como plantean Gutiérrez y Prieto (2004) "(...) da sentido a lo que hacemos, incorpora mi sentido al sentido de la cultura y del mundo, comparte y da sentido, relaciona y contextualiza experiencias, relaciona y contextualiza discursos e impregna de sentido las diversas prácticas y la vida cotidiana" (p. 18)

Todo esto constituye un marco socio-cultural en el que la bioética pueda dar luces para la toma decisiones, pues como lo afirma Jiménez (2008) “(...) no se pueden separar los dilemas morales de sus contextos, sean estos institucionales, políticos, económicos, sociales o culturales." (pp. 245-246). Diferentes dilemas bioéticos aparecen producto del contacto entre visiones émicas (la de las poblaciones) y la de la biomedicina. Son situaciones en las cuales las creencias, los significados y los comportamientos de poblaciones americanas ponen en riesgo la salud de sus individuos debido a que favorecen la presencia de las parasitosis intestinales en los mismos. Sin embargo, debido a que están firmemente arraigados en su cultura y en su manera de pensar, 
son difíciles de abordar desde la biomedicina. Y más difícil aún es el tratar de cambiar estas ideas sin influir o afectar de manera significativa su cultura y sus costumbres en general. Además, pueden constituir puntos de quiebre que pueden llevar al fracaso de estrategias antiparasitarias que no tengan en cuenta previamente esas concepciones, por ser muchas veces antagónicos a los conceptos biomédicos.

Como señalan Gordon y Levin, cuando personas de distintas culturas interactúan se generan diferentes suposiciones, por ejemplo creer que su modo de ver las cosas es el más válido. De allí pueden surgir confusiones, dilemas bioéticos y conflictos de valores (2007). En ese sentido, "(...) la etnografía es un método excelente para examinar supuestos culturales y para el estudio de sus efectos en los comportamientos, interacciones sociales, y las decisiones en el ámbito sanitario." (Traducción de Gordon y Levin, 2007, p. 57).

\section{CONCLUSIONES}

La aproximación émica faculta entender otras lógicas que la biomedicina no reconoce como tales, pero que por supuesto son importantes desde la concepción antropológica y bioética. Los conceptos de illness, disease y sickness permiten identificar diferencias socioculturales en la lectura de las parasitosis intestinales $y$ en las acciones que se deriven ante ellas, por ello

debería conllevar a un replanteamiento sobre cómo abordarlas desde la biomedicina de una manera dialógica y bioética, lo que implica asumir una postura respetuosa de las diferencias, con el fin de construir estrategias educativas y de control en que los pueblos y los profesionales de la salud aporten lo positivo de cada uno desde un plano de interpretación intercultural. Ello supondría no hacer mofa de, ni desconocer, otras construcciones socio-culturales sobre las patologías, pues tal cosa podría avergonzar a la gente y hacer que se alejara de los servicios de salud.

Retomando el tema de la percepción de la enfermedad, cuando los parásitos intestinales son reconocidos como causantes de un padecimiento por parte de los saberes de las poblaciones el trabajo de los profesionales de la salud puede tornarse menos arduo. Sin embargo, cuando son calificados de inocuos o necesarios para ciertos procesos, como la digestión, tal como apareció en algunos de los casos citados, las labores de prevención y educación se pueden complicar, pues implican involucrarse en el concepto de normalidad, que tiene una carga valorativa. Lo normal no es culturalmente homogéneo; al encontrarse normalidades distintas se puede producir un conflicto sobre cuál de las dos ha de regir y generar una lucha de poderes, enraizada en los saberes. Los sistemas oficiales de salud acatan la normalidad biomédica, pero los sujetos imbuidos en culturas médicas distintas tenderán a reconocer la propia y actuar de acuerdo a ella. Por eso, conocer las perspectivas émicas sobre potenciales o efectivos problemas de salud constituyen un deber de ética profesional de quienes trabajan en salud pública.

Por otra parte, de lo analizado se deduce que todo grupo social construye prácticas preventivas cuando percibe como nocivos los parásitos intestinales, que bien pueden ser fruto del conocimiento tradicional o producto de una mezcla o reinterpretación de distintos saberes, entre ellos el biomédico. El sistema preventivo que se elabore dará pie al actuar de la gente, en consonancia con la prioridad que se le asigne al problema. Por ende, entender las prácticas preventivas de los pueblos ayudará a diseñar y desarrollar programas de prevención, promoción y educación en salud acordes a las culturas de los pueblos.

Asimismo, la construcción sociocultural del cuerpo (cómo los saberes populares y tradicionales construyen la anatomía) se relaciona con la interpretación de los parásitos intestinales, que tiene en cuenta el momento que vive el sujeto y su relación con el ambiente para cimentar el razonamiento que explica los efectos percibidos. Es frecuente que las personas reconozcan los parásitos por el lugar del cuerpo en que se piensa que viven (usualmente en el intestino y en el estómago) y por lo que hacen dentro del organismo. Concepciones culturales del cuerpo distintas entre nativos y personal de salud generarán dificultades para el control y tratamiento de las parasitosis.

Ocasionalmente la gente reconoce síntomas de las parasitosis intestinales, pero no las patologías en sí mismas. A veces se las percibe, pero no le dan importancia y otros las consideran potencialmente serias para la salud. Prevenir o educar sobre patologías que la gente no percibe o que no les da relevancia se convierte en una tarea titánica, si no infructuosa en la medida que el personal de salud no se adentra en la Cultura de los pueblos. Dado que la bioética reconoce a las personas como sujetos de derechos, entre los cuales están el de 
la libertad y el respeto a la diferencia, entender las perspectivas émicas sobre las enfermedades es un deber de los profesionales para poder planificar y prestar de una mejor manera los diversos servicios de salud. También lo es para entender las decisiones que el usuario toma respecto a su salud. Es así que estudiar las culturas, antes de trabajar en poblaciones diferentes a la propia, tendría que constituirse en un deber bioético.

El tipo de prácticas preventivas depende del tipo de conocimientos que los pueblos hayan incorporado y de la percepción de anormalidad del padecimiento que se analice. Además, algunas construcciones sociales de las parasitosis intestinales y valoraciones distintas de lo que la biomedicina considera sus factores de riesgo, junto con las dificultades para efectuar cambios en las condiciones sanitarias, aumentan la exposición a estas enfermedades. Sin embargo, es dable preguntarse si ¿eso justifica utilizar sólo la visión de la biomedicina para abordar los problemas de salud? Los resultados muestran que no. Allí la Bioética tiene un papel importante. Ella debe estar presente en los procesos de planificación de trabajos (aplicados o dirigidos a generar conocimiento) desde el principio, con el propósito de evitar etnocentrismos, por lo cual es fundamental partir tanto de las necesidades de la gente como de sus saberes, de sus miradas e interpretaciones. Ello permitirá comprender realidades y, a partir de ahí, agentes de salud y poblaciones tomar decisiones en conjunto. Si no es así, y las decisiones provienen de agentes externos, de manera vertical, habría que cuestionarse si desde la ética profesional esto es respetuoso de los sujetos, así la toma de decisiones esté guiada por la buena voluntad.

Contactos con saberes de otras culturas y su subvaloración o desconocimiento por parte de la biomedicina acarrearán tanto dificultades de comunicación como posibles fracasos en actividades y programas de salud. Para evitar esto se recomiendan capacitaciones en ciencias sociales, con una mirada bioética, para que el personal de salud pueda plantear políticas y actividades de atención, prevención y promoción de la salud culturalmente adaptadas y aceptadas por la población con quien trabajan.

En virtud de lo anterior, se recomienda que las universidades ofrezcan a los estudiantes de las profesiones de la salud asignaturas que discutan y analicen la construcción socio-cultural de las patologías y el cuerpo humano; los efectos de las diferencias socio-culturales tanto en la prevención de la enfermedad como en la atención y la promoción de la salud y la relación de lo anterior con los Derechos consagrados en la Constitución Política de un país pluriétnico y multicultural, como lo es Colombia. Materias como Antropología Médica, Antropología de las Enfermedades Infecciosas, Antropología Médica Aplicada y Bioética compartidas, de manera interdisciplinaria, por docentes con perspectivas profesionales distintas, aportarían al análisis crítico de las dinámicas socio-biológicas de las patologías (como las parasitosis intestinales) y de los derechos correlativos de los usuarios en los servicios de salud. A nivel de profesionales de la salud se sugiere hacer talleres de capacitación, antes de trabajar con poblaciones culturalmente distintas, mediante el análisis de casos, en que se discutan los dilemas bioéticos que pueden surgir al interactuar con dichas poblaciones y los posibles caminos a seguir para la toma de decisiones en salud.

Partir de perspectivas émicas y contar con una efectiva participación comunitaria constituye una manifestación de respeto al Otro, al mismo tiempo que facilitará co-construir nuevo conocimiento que permite abordar los problemas de salud de manera más exitosa, sin imposiciones, sino entendiendo la lógica de las partes involucradas. Asumir a las poblaciones como objetos de decisiones externas, además de constituir un problema bioético, puede acarrear la no reducción de las prevalencias de las parasitosis intestinales.

Asimismo, hay que reconocer también que el análisis de la visión émica, aunque es fundamental, no lo es todo. En el problema de las parasitosis intestinales intervienen también condiciones infraestructurales, como la deficiencia en servicios públicos, que conllevan situaciones conflictivas para los profesionales de la salud, como agentes externos a las comunidades. Es decir, se pueden hacer campañas de educación que efectivamente mejoren la prevención, pero realidades socio-económicas (por ejemplo, pobreza, participación comunitaria, empoderamiento,) y políticas (compromisos y acciones de líderes y gobiernos a diferentes niveles) pueden impedir que lo que se aprendió surta el efecto deseado o que se modifiquen las condiciones estructurales que generan los problemas. En este punto se generan problemas morales que deberían ser analizados por la bioética.

Finalmente, se propone entonces fortalecer el papel de la bioética como guardiana de la diversidad, evitando que se presenten posturas hegemónicas 
alrededor del manejo del conocimiento y de las prácticas en salud, respetando el conocimiento, prácticas y costumbres locales, pero generando un diálogo y una intermediación con la biomedicina y el sistema de salud imperante. Esto se puede lograr, como ya se esbozó anteriormente, por medio de un trabajo interdisciplinar, de una capacitación del personal de salud en el análisis bioético, y por medio de un diálogo constante y continuo con las comunidades, que genere una apropiación y empoderamiento de las problemáticas por parte de la población.

\section{REFERENCIAS}

- AGUDELO-FLOREZ, P., RESTREPO, B.N., Y PALACIO, L.G. (2009). [Knowledge and practices concerning taeniasis-cysticercosis in Colombian pig-breeders]. Rev Salud Publica (Bogota), 11(2), 191-199.

- AgudELO-LOPEZ, S., GÓMEZ-ROdRÍGUEZ, L., CORONADO, X., OROZCO, A., VALENCIA-GUTIERREZ, C.A., RESTREPO-BETANCUR, L.F., ... BOTERO-PALACIO, L.E. (2008). Prevalencia de Parasitosis Intestinales y Factores Asociados en un Corregimiento de la Costa Atlántica Colombiana. Revista de Salud Pública, 10, 633-642.

- ARRAtia, A. (1999). Bioética y toma de decisiones en Enfermería. Cuadernos de Bioética, 39 (3): 512-521. http://aebioetica. org/revistas/1999/3/39/512.pdf

- Asociación de Bioética de la Comunidad de Madrid (2012). ¿Qué es la Bioética? ¿Para qué sirve? España. http://www. abimad.org/documentaci\%C3\%B3n-por-temas/1-bio\%C3\% A9tica-general-y-deontolog\% C3\%ADa/bio\% C3\% A 9 tica-una-nueva-definici\%C3\%B3n/

- Botero, D., y RESTREPO, M. (2005). Conceptos Generales sobre Parasitología. Parasitosis Humanas (4 ed., pp. 2-27). Medellín, Colombia: Corporación para Investigaciones Biológicas.

- CASTRO, E., CALDAS, L., FLÓREZ, L.P., MOTTA, L.Y., y Vargas, Y.P. (2008). Prácticas, creencias y actitudes en torno a las parasitosis intestinales en el asentamiento "San Francisco" de Popayán. Revista Facultad Ciencias de la Salud, Universidad del Cauca, 10(2), 39-47.

- DíAZ, M.P. (2002). El riesgo en salud: entre la visión del lego y el experto. Una perspectiva sociocultural. Bogotá, Colombia: Unibiblos, Universidad Nacional de Colombia.

- DÍAZ, M., MONCADA, L. I., REYES, P., FERNÁNDEZ, J. A., CANO, D. F., y SUÁREZ, R. F. (2010). Conocimientos, actitudes y prácticas sobre las geohelmintiasis en una comunidad rural de Colombia. Revista Facultad de Medicina de la Universidad Nacional de Colombia, 18 (1), 12-22.

- EL KATSHA, S., y WATTS, S. (1997). Schistosomiasis in two Nile delta villages: an anthropological perspective. Tropical Medicine y International Health, 2(9), 846-854. doi: 10.1046/ j.1365-3156.1997.d01-409.x

- ESCOBAR, M.T. (2009). Consentimiento informado en odontología y sus especialidades (Feriva SA Ed.). Colombia: Feriva SA.

- ESCOBEDO, A. A., ALMIRALL, P., ALFONSO, M., AVILA, I., ClMERMAN, S., SALAZAR, Y., ... GARCIA, R. M. (2011). Caregiver perspectives for the prevention, diagnosis and treatment of childhood giardiasis in Havana City, Cuba. A qualitative study. Acta Tropica, 119(2-3), 99-106. doi: 10.1016/j.actatropica.2011.04.014

- ESCOBEDO, A. A., Y CIMERMAN, S. (2007). Giardiasis: a pharmacotherapy review. Expert Opinion on Pharmacotherapy, 8(12), 1885-1902. doi: 10.1517/14656566.8.12.1885

- FENG, Y., Y XIAO, L. (2011). Zoonotic potential and molecular epidemiology of Giardia species and giardiasis. Clinical Microbiology Reviews, 24(1), 110-140. doi: 10.1128/cmr.00033-10

- FERNÁNDEZ-NIÑO, J.A., REYES-HARKER, P., MONCADAÁLVAREZ, L.I., LÓPEZ, MYRIAM C., CHÁVES, M.P., KNUDSON, A., y ARIZA, Y. (2007). Tendencia y Prevalencia de las Geohelmintiasis en La Virgen, Colombia 1995-2005. Revista de Salud Pública, 9, 289-296.

- GAMBOA, M.I., BASUALDO, J.A., KOZUBSKY, L., COSTAS, E., CUETO RUA, E., y LAHITTE, H.B. (1998). Prevalence of intestinal parasitosis within three population groups in La Plata, Argentina. European Journal of Epidemiology, 14(1), 55-61. doi: 10.1023/A:1007479815249

- GAZZINELLI, M.F., KLOOS, H., DE CASSIA MARQUES, R., DOS REIS, D.C., y GAZZINELLI, A. (2008). Popular beliefs about the infectivity of water among school children in two hyperendemic schistosomiasis areas of Brazil. Acta Trop, 108(23), 202-208. doi: 10.1016/j.actatropica.2008.05.009

- GEERTZ. (1994). Conocimiento Local (1 ed.). Barcelona, España: Ediciones Paidós Ibérica.

- Gil nebot, M.Á., estrada BAllesteros, C., PIRES ALCALDE, M.L., y AGUIRRE MARTíN-GIL, R. (2002). La investigación cualitativa y la promoción de la salud en la Comunidad de Madrid. Revista Española de Salud Pública, 76, 451-459.

- GÓES, V., FOCESI, M., RIBEIRO, N., y L'ABBATE, S. (2000). Promoción de la Salud. Promotion y Education: International Journal of Health Promotion and Education VII(4), 8-12.

- GORDON, E.J., y LEVIN, M.W. (2007). Contextualizing ethical dilemmas: Ethnography for bioethics (Vol. 11, pp. 83-116): Emerald Group Publishing Limited.

- GUTIÉRREZ, F., y PRIETO, D. (2004). Mediación Pedagógica. Santiago: Ediciones La Copia Fiel.

- HOTEZ, P.J., BRINDLEY, P.J., BETHONY, J.M., KING, C.H., PEARCE, E.J., y JACOBSON, J. (2008). Helminth infections: the great neglected tropical diseases. The Journal of Clinical Investgation, 118 (4), 1311-1321. doi: 10.1172/jci34261

- HUGHES, C. (1975). Etnomedicina. Enciclopedia Internacional de las Ciencias Sociales (pp. 578-582). Madrid: Editorial Aguilar.

- JIMÉNEZ, M. (2008). Encuentros entre bioética y antropología. Paper presented at the Miradas, encuentros y críticas antropológicas, congreso de antropología de la FAAEE. Gipuzkoa, España

- KLINE, K., MCCARTHY, J.S., PEARSON, M., LOUKAS, A., y HOTEZ, P.J. (2013). Neglected tropical diseases of Oceania: review of their prevalence, distribution, and opportunities for control. PLoS Neglected Tropical Diseases, 7 (1), e1755. doi: 10.1371/journal.pntd.0001755

- KLOOS, H. (1995). Human behavior, health education and schistosomiasis control: a review. Soc Sci Med, 40(11), 1497-1511.

- KRUMEICH, A., WEIJTS, W., REDDY, PR., y MEIJER-WEITZ, A. (2001). The benefits of anthropological approaches for health promotion research and practice. Health Education Research, 16(2), 121-130. doi: 10.1093/her/16.2.121

- LANE, S., y LLOYD, D. (2002). Current trends in research into the waterborne parasite Giardia. Critical Reviews in Microbiology, 28 (2), pp. 123-147. doi: 10.1080/1040-840291046713 
LEAH, Q. (1999). Helminthic infection and anemia in children: A comparative analysis of western and traditional medicine: Tamanco jurisdiction, Napo river, Amazon. Lambda Alpha Journal, 29, 29-39. http://etnobotanica.us/wp-content/uploads/2011/12/ lambda-alpha-jounral-1999.pdf

- LEJARRAGA, A. (2004). La construcción social de la enfermedad. Archivos Argentinos de Pediatría, 102 (4), 271-276.

- LEÓN, F. (2011). Bioética, Salud Pública y Educación para la Salud. Revista de Bioética Latinoamericana, 7 (1): 7-18. http:// www.saber.ula.ve/bitstream/123456789/32747/3/articulo2.pdf

- LIND, J.D. (2009). The political ecology of intestinal parasites among Nicaraguan immigrants in Monteverde, Costa Rica. (Ph.D.), University of South Florida, Florida, E.U. Retrieved from http://scholarcommons.usf.edu/etd/2062/

- LOLAS, F. (2007). Estudios de Bioética Social. Prioridades en Salud y Salud Intercultural. En Lolas, S, Fernando., Douglas K., Martin y Quezada, Álvaro Eds. Estudios de bioética social prioridades en salud y salud intercultural. (pp. 15 -27). CIEB, Universidad de Chile, Centro Colaborador en Bioética OMS. Primera edición, Chile. http://www.uchile.cl/bioetica/

- LOZANO, R., NAGHAVI, M., FOREMAN, K., LIM, S., SHIBUYA, K., ABOYANS, V., . . MEMISH, Z.A. (2012). Global and regional mortality from 235 causes of death for 20 age groups in 1990 and 2010: a systematic analysis for the Global Burden of Disease Study 2010. The Lancet, 380(9859), 2095-2128. doi: 10.1016/s0140-6736(12)61728-0

- LUSTIGMAN, S., PRICHARD, R.K., GAZZINELLI, A., GRANT, W.N., BOATIN, B.A., MCCARTHY, J.S., y BASANEZ, M.G. (2012). A research agenda for helminth diseases of humans: the problem of helminthiases. PLoS Neglected Tropical Diseases, 6(4), e1582. doi: 10.1371/journal.pntd.0001582

- MARTínEZ A. (2008). Teorías sobre la cultura, el poder y la enfermedad (1 ed.). Barcelona: Anthropos.

- MORENO-ALTAMIRANO, L. (2007). Reflexiones sobre el trayecto salud-padecimiento-enfermedad-atención: una mirada socioantropológica. Salud Pública de México, 49, 63-70.

- OlSEN, A., VAN LIESHOUT, L., MARTI, H., POLDERMAN, T., POLMAN, K., STEINMANN, P., . . . MAGNUSSEN, P. (2009). Strongyloidiasis--the most neglected of the neglected tropical diseases? Transactions of the Royal Society of Tropical Medicine and Hygiene, 103 (10), 967-972. doi: 10.1016/j. trstmh.2009.02.013

- OPS (2007). Control de las helmintiasis en los países del Caribe inglés y francés: Hacia la Resolución 54.19 de la Asamblea Mundial de la Salud. http://www1. paho.org/Project.asp?SEL=T PyLNG=SPAyID=126yPRGRP=docs_gen

- OPS, BID, y Instituto de Vacunas Sabin. (2011). Un lamado a la acción: hacer frente a los helmintos transmitidos por el contacto con el suelo en Latinoamérica y el Caribe (p. 33). Retrieved from http://www.paho.org/equity/index2.php?option= com_docmanytask=doc_viewygid $=46$ yltemid $=$

- PAHO. (2009). Epidemiological profiles of neglected diseases and other infections related to poverty in Latin America and the Caribbean. http://new.paho.org/hq/index.php?option=com_co ntentyview $=$ articleyid $=1247 \% 3$ Aepidemiological-profiles-ofneglected-diseases-and-other-infections-related-to-povertyin-lacycatid $=3722 \% 3$ Ahsd0108e-neglected-d-statistics-andmapsyltemid $=4030$ ylang $=p t$

- PEBlEY, A., HURTADO, H., y GOLDMAN, N. (1996). Beliefs about children's illness among rural Guatemalan women (p. 52). Retrieved from http://www.rand.org/pubs/drafts/DRU1448.html
PEDRAZZANI, E.S., MELLO, D.A., PIZZIGATTI, C.P., y BARBOSA, C.A.A. (1990). Aspectos educacionais da intervenção em helmintoses intestinais, no subdistrito de Santa Eudóxia, Município de São Carlos - SP. Cadernos de Saúde Pública, 6, 74-85.

- PÉREZ SERRANO, G. (2004). Pedagogía social y educación social, construcción científica e intervención práctica. (2 ed.). Madrid: DE Ediciones.

- PÉREZ SERRANO, G. Pedagogía social y educación social, Construcción científica e intervención práctica. Narcea, S.A. DE Ediciones. Madrid, 2004.

- PINZÓN, C., y SUÁREZ, R. (1992). Las mujeres lechuza. Bogotá: ICAN/Colcultura/CEREC.

- QUINLAN, M.B., QUINLAN, R.J., y NOLAN, J.M. (2002). Ethnophysiology and herbal treatments of intestinal worms in Dominica, West Indies. Journal of Ethnopharmacology, 80 (1), 75-83.

- RAMíREZ, N.A., DÍAZ, M.P., REYES, P., y CUECA, O. (2011). Educación lúdica: Una opción dentro de la educación ambiental en salud, seguimiento de una experiencia rural colombiana sobre las geohelmintiasis. Revista Facultad de Medicina de la Universidad Nacional de Colombia, 19 (1), 23-36.

- SANZÓN, F., VELA, J.C., VALENCIA, H.F., y MONTENEGRO, L. (1999). Una estrategia antiparasitaria original en Arboleda, Nariño. Colombia Médica, 30 (3), 112-117.

- SARTI, E., FLISSER, A., SCHANTZ, P. M., GLEIZER, M., LOYA, M., PLANCARTE, A., WIJEYARATNE, P. (1997). Development and evaluation of a health education intervention against Taenia solium in a rural community in Mexico. Am J Trop Med Hyg, 56(2), 127-132.

- SESIA, P. (1999). Los padecimientos gastrointestinales entre los chinantecos de Oaxaca: aspectos denotativos y connotativos del modelo etnomédico Alteridades, 9 (17), 71-84.

- STANLEY, S.L., JR. (2003). Amoebiasis. The Lancet, 361(9362), 1025-1034. doi: 10.1016/s0140-6736(03)12830-9

- SY, ANAHI. (2009). Una revisión de los estudios en torno a enfermedades gastrointestinales. En busca de nuevas alternativas para el análisis de los procesos de salud-enfermedad. Salud Colectiva, 5 (1), 49-62.

- UTZINGER, J., Y KEISER, J. (2004). Schistosomiasis and soiltransmitted helminthiasis: common drugs for treatment and control. Expert Opinion on Pharmacotherapy, 5 (2), 263-285. doi: 10.1517/14656566.5.2.263

- VILLADIEGO, V. (1995). El bacteriólogo: más allá del microscopio. Revista Salud Culturas de Colombia, 13, 7-10.

- WENZEL GEISSLER, P. (1998). 'Worms are our life', part I: Understandings of worms and the body among the Luo of western Kenya. Anthropology y Medicine, 5 (1), 63-79. doi: $10.1080 / 13648470.1998 .9964549$

- WHO (2005). Report of the third global meeting of the partners for parasite control. http://docs.watsan.net/Downloaded_Files/ PDF/WHO-2005-Report.pdf

- WIKMAN, A., MARKLUND, S., Y ALEXANDERSON, K. (2005). Illness, disease, and sickness absence: an empirical test of differences between concepts of ill health. Journal of Epidemiology and Community Health, 59 (6), 450-454. doi: 10.1136/ jech.2004.025346

- ZONTA, M.L., NAVONE, G.T., y OYHENART, E.E. (2007). Parasitosis intestinales en niños de edad preescolar y escolar: situación actual en poblaciones urbanas, periurbanas y rurales en Brandsen, Buenos Aires, Argentina. Parasitología Latinoamericana, 62, 54-60. 